\title{
Thyroid scintigraphy in the management of benign thyroid disease
}

\section{Yaw A. Amoako ${ }^{1}$, Emmanuel N.B. Hammond ${ }^{2,3}$, Awo Assasie-Gyimah ${ }^{2}$ and Osei Sarfo-Kantanka ${ }^{1}$}

Ghana Med J 2018; 52(4): 242-248 doi: http://dx.doi.org/10.4314/gmj.v52i4.12

\author{
${ }^{1}$ Department of Medicine, Komfo Anokye Teaching Hospital, Kumasi, Ghana \\ ${ }^{2}$ Nuclear Medicine Service, Korle Bu Teaching Hospital, Accra, Ghana \\ ${ }^{3}$ Ghana Atomic Energy Commission, Accra, Ghana
}

\author{
Corresponding author: Dr Yaw Ampem Amoako \\ Conflict of interest: None declared \\ E-mail: yamoako2002@yahoo.co.uk
}

\begin{abstract}
SUMMARY
Thyrotoxicosis as a clinical entity has varied aetiologies. Accurate distinction of the underlying aetiology is important to guide therapy. Clinical findings are not always accurate in identifying the underlying aetiology. There is considerable overlap between the various aetiologies when using serum T3/T4 ratio. Although ultrasound is widely available and has reasonable sensitivity, it is operator dependent and misses many cases of early Grave's disease. $99 \mathrm{mTc}$ scintigraphy is a simple and accurate modality for assessing the functional status of the thyroid gland and delineating the aetiology of thyrotoxicosis. This paper seeks to inform on the use of thyroid scintigraphy in the management of benign thyroid disorders.
\end{abstract}

Funding: None

Keywords: Scintigraphy, thyroid gland, functional imaging, Grave's disease, Ghana

\section{INTRODUCTION}

The thyroid gland, a small but important endocrine organ, which is usually located in the anterior neck, functions to synthesise, store and secrete the thyroid hormones thyroxine and tri-iodothyronine. Thyroid hormones are essential for metabolism and act to increase oxygen consumption and basal metabolic rate. ${ }^{1,2}$ SarfoKantanka et al have reported that thyroid disorders are the second leading cause of endocrine morbidity in central Ghana with hyperthyroidism accounting for $9.2 \%$ of all cases seen in their endocrine service. ${ }^{3}$ The same authors have reported that toxic multinodular disease is more common than thyrotoxicosis from diffuse goitre in Ghana. ${ }^{4}$ In this paper, we aim to highlight the essential role of thyroid scintigraphy in the management of benign thyroid disease and alert clinicians in Ghana to the availability of this resource.

\section{The physiologic basis for thyroid scintigraphy}

Anatomic imaging modalities such as ultrasonography or computed tomography (CT) provide information on the architectural structure of the thyroid gland. Ultrasonography is a cost-effective, non-invasive, portable, and safe imaging modality in the evaluation of thyrotoxicosis for detection of non-palpable thyroid cancers that may elude identification on physical examination and guide selection of nodules for fine needle aspiration and biopsy. ${ }^{5-8}$ The scintigraphic appearance however reflects the functional state of the entire thyroid gland as well as the functional status of any nodule (low uptake vs high uptake or same uptake as rest of the thyroid gland) which may have been identified by physical examination or on anatomic imaging modalities such as ultrasonography or computed tomography. ${ }^{9}$ On thyroid scintigraphy, the entire gland may have high uptake of tracer reflecting hyper-function of the gland (eg Grave's disease as shown in Figure 1 or toxic nodular goitre as shown in Figure 2 and Figure 3) or have low uptake of tracer (as occurs in thyroiditis).

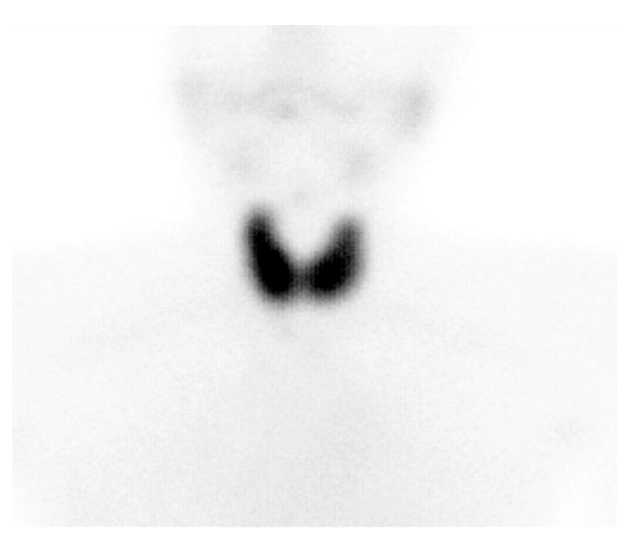

Figure 130 -year-old female diagnosed with hyperthyroidism referred for ablation. TSH $<0.01 \mathrm{mmol} / 1$, FT $4=17.3 \mathrm{pmol} / \mathrm{ml}$, FT3 $=7.0 \mathrm{pmol} / \mathrm{ml}$. Scan showed marked homogeneous increased uptake of pertechnetate consistent with Grave's disease. 


\section{Special Article}

Figure 2 76-year-old female, known with hyperthyroidism and been on carbimazole for 6 years. TSH $<0.01 \mathrm{mmol} / 1$. Thyroid scan showed a TMNG as demonstrated by the bulky thyroid gland with increased inhomogeneous uptake of tracer and a large cold nodule in the left lobe. This nodule was confirmed on ultrasound guided FNA to be non-malignant. She received RAI treatment with $30 \mathrm{mCi}$ I-131 post FNA.

However, nodules within the thyroid gland may exhibit uptake which differs from the remainder of the gland. For instance, in a patient with toxic multinodular goitre there may be a particular nodule with low tracer uptake ('cold' nodule) relative to other nodules in the gland but the entire gland will have high measured uptake (this is the situation of a hypo-functioning or cold nodule within the setting of a toxic multi-nodular gland as demonstrated in Figure 2). Conversely, there may be a dominant hyper-functioning or 'hot' nodule in a patient with multinodular disease (see Figure 3).

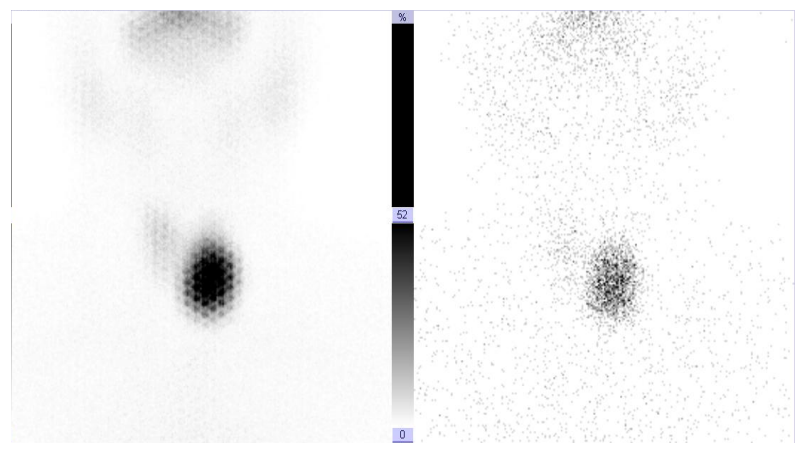

Figure 3 38-year-old female hyperthyroid patient on carbimazole. Her laboratory tests showed TSH $=0.02 \mathrm{mmol} / 1$, FT4 $=11.27 \mathrm{pmol} / 1$. Scan findings revealed a toxic solitary nodule in the left thyroid lobe with suppression of uptake in the remainder of the gland.

The discipline of Nuclear Medicine has long had an essential role in the diagnosis and management of thyroid disorders. The Society of Nuclear Medicine and Molecular Imaging ${ }^{10,11}$ has issued guidelines for the performance of thyroid scintigraphy.
The American Thyroid Association (ATA) and the American Association of Clinical Endocrinologists (AACE) have also issued management guidelines for thyrotoxicosis and other causes of hyperthyroidism. ${ }^{12}$

These guidelines ${ }^{10,12}$ all detail the essential role of nuclear medicine/ thyroid scintigraphy in the management of benign thyroid disorders/ hyperthyroidism.

The applications of thyroid scintigraphy include:

- Evaluation of the general structure of the thyroid gland (e.g. nodular or diffuse enlargement) relative to its function. This may be useful in the differential diagnosis of hyperthyroidism, i.e. distinguishing Graves' disease from toxic nodular goitre.

- Correlation of thyroid palpation with scintigraphic findings to determine the degree of function in a nodule that is palpable or found incidentally at a non-nuclear imaging procedure

- Evaluation of the underlying aetiology of congenital hypothyroidism (total agenesis or hemiagenesis, dyshormonogenesis, incomplete thyroid descent)

- Location of ectopic thyroid tissue (e.g., lingual, incomplete thyroid descent) and evaluation of a neck or substernal mass to confirm that a substernal mass is functioning thyroid tissue.

- Differentiation of thyroiditis (i.e. viral, autoimmune) and factitious hyperthyroidism from Graves' disease and other forms of hyperthyroidism.

- Selection of thyrotoxic patients that qualify for radioactive iodine ablation therapy: Patients with high tracer uptake on thyroid scintigraphy are potential candidates for ablation with radioiodine. In such patients, radioactive iodine uptake studies also can be used in estimating dose of I-131 for therapy if using the calculated dose regime.

The recommended radiopharmaceuticals for thyroid scintigraphy are Technetium $99 \mathrm{~m}$ pertechnetate (99m Tc pertechnetate) and iodine $-123\left({ }^{123} \mathrm{I}\right)$. Radioiodine uptake (RAIU) measures the percentage of administered radioiodine that is concentrated into thyroid tissue after a fixed interval, typically 24 hours. A technetium uptake measures the percentage of administered technetium that is trapped by the thyroid after a fixed interval, usually 20 minutes. ${ }^{99 \mathrm{~m}} \mathrm{Tc}$ pertechnetate has a similar size and charge as iodide. It is taken up in the same manner via active transport mechanisms by the sodium iodide symporter (NIS) located in the basolateral membrane of the thyroid follicular cells which enables the thyroid gland to concentrate iodide by a factor of $20-40$ times greater than its plasma levels. ${ }^{13-15}$ Unlike iodide however, pertechnetate after its trapping does not undergo organification. ${ }^{2,16}$ 


\section{Special Article}

Apart from the thyroid gland, NIS is also expressed on choroid plexus, salivary glands, gastric mucosa, lactating mammary glands and ciliary body of eyes. Technetium $99 \mathrm{~m}$ pertechnetate is readily available from a Molybdenum-99/ Tc-99m generator which is usually available within all nuclear medicine departments. It is cheap and allows for imaging to be completed within a shorter time. Iodine-123 (I-123) on the other hand is cyclotron produced and must be pre-ordered from a distributor. Iodine-123 has an advantage in the identification of discordant nodules - nodules which appear warm on pertechnetate images but have decreased uptake or appear cold on I-123 imaging. Such discordant nodules have the capacity to trap pertechnetate but cannot organify iodine and are therefore regarded as true cold nodules with a higher risk of malignancy. ${ }^{2,9}$ At our centre, ${ }^{99 \mathrm{~m}} \mathrm{Tc}$ sodium pertechnetate is used for thyroid scintigraphy and we recommend biopsy for histologic correlation of all 'cold' nodules and those with suspicious features on ultrasonography.

\section{Thyroid scintigraphy in differential diagnosis of thy- rotoxicosis}

In thyrotoxicosis, the identification of the specific underlying aetiology is useful in guiding the formulation of a therapy plan. Grave's disease is a stimulationinduced thyrotoxicosis while thyroiditis is a destructioninduced thyrotoxicosis. Hyperthyroidism associated with normal to elevated radioactive iodine uptake (see Table 1) as occurs in Grave's disease, toxic adenoma or toxic multinodular goitre, may be treated with antithyroid drugs, radioactive iodine therapy, and thyroidectomy.

Table 1 Aetiologies of thyrotoxicosis and uptake of tracer on thyroid scintigraphy

\begin{tabular}{|l|l|}
\hline Elevated/normal uptake & Low/ near absent uptake \\
\hline Grave's disease & Painless thyroiditis \\
\hline Toxic adenoma & Subacute/ deQuervain's thyroiditis \\
\hline Toxic multinodular goitre & Factitious/ ingestion of thyroxine \\
\hline $\begin{array}{l}\text { TSH producing pituitary } \\
\text { adenoma }\end{array}$ & Amiodarone induced thyroiditis \\
\hline
\end{tabular}

Treatment options for thyroiditis (low radioactive iodine uptake) induced thyrotoxicosis include beta $(\beta)$ blockers to relieve symptoms and glucocorticoids to relieve inflammatory effects, if present.

A radioactive iodine uptake and scan should be performed when the cause of a patient's thyrotoxicosis cannot be definitively determined by history and physical examination. ${ }^{12,17,18}$ Treating painless thyroiditis as if it were Grave's disease would be completely inappropriate. ${ }^{12,17}$ Painful subacute thyroiditis is caused by viral infection and is characterized by fever and thyroid pain and this usually distinguishes this entity.
However, the clinical presentation alone may not accurately resolve differentiation of the aetiology of thyrotoxicosis.

The symptoms of painless thyroiditis can mimic those of early onset or recurrence of Grave's disease, and this may lead to a mistreatment. The characteristic Grave's ophthalmopathy, dermopathy and thyroid acropachy may not be present in the earlier stages of the disease. ${ }^{19}$ Occasionally, Grave's disease may co-exist with thyroiditis and may develop following the complete remission of Grave's disease, and painless thyroiditis can be followed by Grave's disease. ${ }^{20-23}$

Diagnostic testing is essential to ascertain the aetiology of thyrotoxicosis if the diagnosis is not apparent based on the clinical presentation and initial biochemical evaluation. The choice is often dependent on available expertise and resources. Available tools to ascertain the underlying aetiology of thyrotoxicosis include ultrasonography to assess thyroidal blood flow, radioactive iodine uptake (RAIU)/ thyroid scintigraphy and measurement of Thyrotropin receptor antibody (TRAb). ${ }^{8,12,18,24-29}$

Thyroid ultrasound is a cheap, convenient, non-invasive and sensitive technique for assessing the underlying aetiology of thyrotoxicosis. In Grave's disease, sonography has a reported high sensitivity of $95 \%$ compared to $97 \%$ for nuclear scintigraphy. ${ }^{25}$ some authors suggest that ultrasound with colour doppler evaluation should be performed as first step in all hyperthyroid patients, and that scintigraphic examination should be limited only to the uncommon cases, where physician's observation, laboratory assays and/or ultrasound are not diagnostic. ${ }^{25}$ Doppler ultrasound can be used to quantify thyroid vascularity and has been reported useful in distinguishing Grave's disease from destructive thyroiditis. ${ }^{30-32}$

Thyrotoxicosis due to Grave's disease is reported to be associated with increased peak systolic blood velocity through the inferior thyroidal artery whereas low flow is seen in destructive thyroiditis. ${ }^{30}$ European thyroid experts consider ultrasonography as the primary imaging modality for thyrotoxicosis because of its ready availability, convenience and ease of use ${ }^{24}$ but this opinion is not shared by experts in the United States of America. ${ }^{12}$

The ratio of free $\mathrm{T} 3$ to free $\mathrm{T} 4$ helps to differentiate Grave's disease from destruction induced thyrotoxicosis. The serum free T3 is considerably higher in Grave's disease rather than thyroiditis. ${ }^{33}$ Sriphrapradang et al ${ }^{34}$ reported that a serum free $\mathrm{T} 3$ to free $\mathrm{T} 4$ ratio of greater than 4.4 also distinguishes Grave's disease from thyroiditis with a sensitivity of $47 \%$ and a specificity of $92 \%$. Other authors reported the FT3/FT4 ratios of patients with painless thyroiditis overlapped with those of patients with Grave's disease and posited that the ratio 


\section{Special Article}

was useful for differentiating between the two disorders when the FT4 values were high. ${ }^{35}$

TRAb measurements may be negative in $5-10 \%$ of Grave's disease patients while some patients with thyroiditis may have positive TRAb assays. ${ }^{26,36,37}$ It has been recommended that a radioactive iodine uptake and scan be performed when the cause of a patient's thyrotoxicosis cannot be definitively determined by history and physical examination. ${ }^{12,17}$

Thyroid scintigraphy with radioiodine uptake measurement (RAIU) is the most reliable modality for distinguishing Grave's disease from thyroiditis. . $^{6,8,12,27-29,38}$ The American Thyroid Association and American Association of Clinical Endocrinologists guidelines from 2011 suggest an approach that recommends measurement of serum TRAb levels when measurement of radioactive iodine uptake or thyroid scintigraphy is not available or contraindicated. ${ }^{12}$ Other authors however endorse using TRAb to identify cases of Grave's disease and disagree with this diagnostic restriction on the use of TRAb assays. 24,39 Yamashita et al have reported that the more frequent use of TRAb assays rather than scintigraphy in Japan compared with the United States and Korea is related to multiple factors including the relative shortage of nuclear medicine facilities. ${ }^{40} \mathrm{~A}{ }^{123} \mathrm{I}$ or ${ }^{99 \mathrm{~m}} \mathrm{Tc}$ pertechnetate scan should be obtained when the clinical presentation suggests a Toxic adenoma (TA) or toxic multinodular goitre (TMNG) ${ }^{18}$

Biochemical and scintigraphic changes in thyroiditis The thyroiditis includes Hashimoto's thyroiditis, painful subacute thyroiditis, painless sporadic thyroiditis and painless postpartum thyroiditis. Hashimoto's thyroiditis, painless sporadic thyroiditis and painless postpartum thyroiditis are all associated with autoimmune phenomena. ${ }^{41}$ The thyroidites are associated with destruction of the thyroid gland with release of preformed thyroid hormone into the circulation results in a transient hyperthyroidism. As the glandular stores of thyroid hormone are depleted, the clinical course of disease is characterised by periods of hypothyroidism and euthyroidism.

The first biochemical change in inflammatory thyroiditis before the onset of thyrotoxicosis is an increase in the serum concentration of thyroglobulin. Similar to other forms of thyrotoxicosis, the serum concentration of thyroid stimulating hormone (TSH) is suppressed, and concentrations of total and free triiodothyronine (T3) and thyroxine (T4) are elevated with serum T4 concentrations proportionally higher than $\mathrm{T} 3$ concentrations, reflecting the ratio of stored hormone in the thyroid gland (whereas in Graves' disease and in toxic nodular goitre, T3 is preferentially elevated).
These changes in hormonal levels are associated with corresponding changes in radiopharmaceutical uptake on scintigraphy. During the early stages of thyroiditis, the serum T4 levels are elevated and TSH is suppressed. This phase is associated with a low tracer uptake on scintigraphy. In the mid phase of thyroiditis, serum T4 is low while TSH and uptake on scintigraphic are high. Normalisation of serum TSH and T4 in the latter stages is associated with a normal uptake on scintigraphy.,41

\section{Amiodarone induced thyrotoxicosis}

Amiodarone, an anti-arrhythmic drug has a high iodine content. Most patients on amiodarone therapy remain euthyroid; however, some may develop thyroid function anomalies which may be hypothyroidism or thyrotoxicosis depending on the iodine status of the patient's locale. Patients from iodine depleted areas usually get amiodarone induced thyrotoxicosis (AIT) while those living in iodine replete areas get amiodarone induced hypothyroidism. ${ }^{42,43}$ There are two distinct pathophysiologic mechanisms for AIT.

While AIT type I is thought to be due to excess iodine mediated increased production of thyroid hormone, AIT type II is the result of a destructive thyroiditis. It is important to distinguish the two types of AIT because their therapy is different. Type I AIT may show normal or increased tracer uptake on scintigraphy but type II AIT is associated with low tracer uptake. Colour flow Doppler may show increased flow/ hypervascularity in cases of type I AIT and absence of hypervascularity in type II AIT. $^{42,44}$

\section{Thyroid scintigraphy in assessment of function of a thyroid nodule}

Correlation of thyroid palpation with scintigraphic findings is important to determine the degree of function in a nodule that is palpable or found incidentally during routine physical examination or at a non-nuclear imaging procedure. Compared to ultrasound, thyroid scintigraphy has a lower sensitivity for diagnosing thyroid nodules ${ }^{24,25}$ due to the limitation posed by the intrinsic resolution of the gamma camera used for nuclear medicine imaging. Most gamma cameras cannot pick- up nodules below $1 \mathrm{~cm}$ in size. ${ }^{2}$

Several guidelines ${ }^{12,18}$ and other authors ${ }^{7,9,24,28}$ recommend the use of thyroid scintigraphy to assess the function of clinically palpable nodules or those found at non-nuclear medicine imaging procedures such as ultrasonography. If a nodule is composed of cells that do not make thyroid hormone (do not absorb iodine/ nonfunctioning), then it will appear "cold" on scintigraphy (see Figure 2). 
A nodule that is producing too much hormone will appear "hot"' (see figure 3). Functional assessment of a thyroid nodule is important as $15-20 \%$ of cold nodules are malignant ${ }^{2}$ with this percentage increasing further in patients who are younger, male, have cervical lymphadenopathy, history of radiation to the neck and family history of thyroid abnormalities. The incidence of malignancy in a cold nodule in multinodular goitres is however very low (less than 5\%) compared to gland with a single nodule. A functioning nodule however is less likely to be malignant with a $1 \%-5 \%$ chance of the hot nodule being malignant. ${ }^{2}$

Ultrasound guided fine needle aspiration (FNA) and cytology is the most accurate test for detecting malignancy in a thyroid nodule. However, FNA is invasive and may produce indeterminate results in up to $25 \%$ of cases $^{45}$. Scintigraphy assessment of nodules reduces the potential number of un-needed biopsies and guides patient management.

Evaluation of the aetiology of congenital hypothyroidism (total agenesis or hemiagenesis, dyshormonogenesis, incomplete thyroid descent)

In countries where screening programmes exist, children with congenital hypothyroidism are usually identified in the neonatal period utilising sensitive TSH assays. Thyroid scintigraphy helps to identify the aetiology or abnormality responsible for the congenital hypothyroidism. Scintigraphy can identify agenesis (absence of uptake), hypoplasia of a gland in situ (with or without hemithyroid), a normal or large gland in situ with or without abnormally high levels of uptake (Figure 4), and an ectopic thyroid at any point along the pathway of the normal embryological descent from the foramen caecum at the base of the tongue to the thyroid cartilage. ${ }^{46,47}$ Combining scintigraphy and thyroid ultrasound in an individual patient helps to improve diagnostic accuracy. ${ }^{46}$

\section{Thyroid scintigraphy prior to radioiodine ablation} In addition to aiding the differentiation of the various aetiologies of hyperthyroidism, thyroid scintigraphy and radioiodine uptake (RAIU) is indicated prior to treatment with iodine -131 . Thyrotoxic patients with high tracer uptake on thyroid scintigraphy are potential candidates for ablation with radioiodine and the dose administered for the treatment may be determined using empiric dosing or a calculated dose.

If the calculated or estimated dosing approach is to be used, the administered dose is determined using multiple parameters which includes the radioiodine uptake measurement. Even in empiric dosing schemes, patients with multinodular goitre tend to be given higher doses than those with Grave's disease.
Scintigraphic imaging directly guides iodine - 131 treatment planning to maximise therapeutic benefit while minimising adverse effects.

Nuclear medicine imaging and therapy in managing benign and malignant thyroid disease is established and is the most successful example of molecular radiotheranostics. $^{48}$

Figure 4 5-year-old boy diagnosed with primary hypothyroidism with 3 months history of thyroxine ingestion. Thyroid scan shows a markedly enlarged thyroid gland with increased inhomogenous uptake of the tracer. The increased tracer uptake in the presence of hypothyroidism is an indication of intact $\mathrm{Na} / \mathrm{I}$ symporters responding to stimulation by high TSH levels as occurs in thyroid dyshormonogenesis.

\section{The experience from the National Nuclear Medicine centre in Ghana}

There is a nuclear medicine service located within the National Radiotherapy and Nuclear Medicine Centre at the Korle-Bu Teaching Hospital. At the centre, thyroid scintigraphy is performed using Technetium $-99 \mathrm{~m}$ sodium pertechnetate. The images included in this paper illustrate the utility of thyroid scintigraphy in the management of benign thyroid disorders in Ghana. With a second nuclear medicine centre poised to be established at the Komfo Anokye Teaching Hospital in Kumasi, this invaluable resource will become even more accessible to clinicians and many more Ghanaians. A total of 155 scans for benign thyroid disorders were performed during the period June 2014 to June 2017.

Nuclear medicine imaging and therapy have established roles in managing benign thyroid disease and is the most successful example of molecular radiotheranostics. In Ghana, the utility of thyroid scintigraphy is hampered by the limited availability of nuclear medicine facilities.

With plans to open new nuclear medicine facilities and increasing physician awareness, there will be opportunities to further leverage thyroid scintigraphy and enhance its utility in the management of benign thyroid disorders in Ghana. 


\section{CONCLUSION}

Thyroid scintigraphy has numerous roles in the management of benign thyroid disorders.

\section{REFERENCES}

1. Crawford ES, Guarasci DT, Larson SA. A survey of thyroid gland scintigraphy. Journal of nuclear medicine technology. 2009;37(3):173-178.

2. Ziessman HA, O'Malley JP, Thrall JH. Nuclear Medicine: The Requisites E-Book. Elsevier Health Sciences; 2013.

3. Sarfo-Kantanka O, Sarfo FS, Ansah EO, Kyei I. Spectrum of Endocrine Disorders in Central Ghana. International journal of endocrinology. 2017; 2017:5470731.

4. Sarfo-Kantanka O, Kyei I, Sarfo FS, Ansah EO. Thyroid Disorders in Central Ghana: The Influence of 20 Years of Iodization. Journal of thyroid research. 2017; $2017: 7843972$.

5. Alzahrani AS, Ceresini G, Aldasouqi SA. Role of ultrasonography in the differential diagnosis of thyrotoxicosis: a noninvasive, cost-effective, and widely available but underutilized diagnostic tool. Endocrine practice: official journal of the American College of Endocrinology and the American Association of Clinical Endocrinologists. 2012;18(4):567-578.

6. Prasek K, Plazinska MT, Krolicki L. Diagnosis and treatment of Graves' disease with particular emphasis on appropriate techniques in nuclear medicine. General state of knowledge. Nuclear medicine review Central \& Eastern Europe. 2015;18(2):110116.

7. Luster M, Verburg FA, Scheidhauer K. Diagnostic imaging work up in multi-nodular goiter. Minerva endocrinologica. 2010;35(3):153-159.

8. Sipos JA, Kahaly GJ. Imaging of thyrotoxicosis. The American journal of medicine. 2012; 125(9):S1-2.

9. Intenzo CM, Dam HQ, Manzone TA, Kim SM. Imaging of the Thyroid in Benign and Malignant Disease. Seminars in Nuclear Medicine. 2012; 42(1):49-61.

10. Balon HR, Silberstein EB, Meier D et al. Society of Nuclear Medicine procedure guideline for thyroid uptake measurement. http://snmmi.files.cmsplus.com/docs/Thyroid $\% 20 \mathrm{U}$ ptake $\% 20$ Measure $\% 20 v 3 \% 200 . p d f$. Accessed on 21 October 2017.

11. Radiology ACo. ACR-SNM-SPR Practice Guidelines for the Performance of Thyroid Scintigraphy and Uptake Measurements. 2009. https://www.acr.org/-/media/ACR/Files/PracticeParameters/thy-scint.pdf?la=en. Accessed on 21 October 2017.

12. Bahn Chair RS, Burch HB, Cooper DS, et al. Hyperthyroidism and other causes of thyrotoxicosis: management guidelines of the American Thyroid Association and American Association of Clinical Endocrinologists. Thyroid. 2011;21(6):593-646.

13. Chung JK. Sodium iodide symporter: its role in nuclear medicine. Journal of nuclear medicine: official publication, Society of Nuclear Medicine. 2002;43(9):1188-1200.

14. De la Vieja A, Dohan O, Levy O, Carrasco N. Molecular analysis of the sodium/iodide symporter: impact on thyroid and extrathyroid pathophysiology. Physiological Reviews. 2000;80(3):1083-1105.

15. Filetti S, Bidart J-M, Arturi F, Caillou B, Russo D, Schlumberger M. Sodium/iodide symporter: a key transport system in thyroid cancer cell metabolism. European Journal of Endocrinology. 1999;141(5):443-457.

16. Meng Z, Zhang G, Sun H, et al. Differentiation between Graves' disease and painless thyroiditis by diffusion-weighted imaging, thyroid iodine uptake, thyroid scintigraphy and serum parameters. Experimental and therapeutic medicine. 2015;9(6):21652172.

17. Pearce EN. Diagnosis and management of thyrotoxicosis. British Medical J. 2006; 332(7554):1369-73.

18. Ross DS, Burch HB, Cooper DS, et al. 2016 American Thyroid Association Guidelines for Diagnosis and Management of Hyperthyroidism and Other Causes of Thyrotoxicosis. Thyroid. 2016;26(10):1343-1421.

19. Bartalena L. Diagnosis and management of Graves disease: a global overview. Nature Reviews Endocrinology. 2013;9(12):724-734.

20. Misaki T, Miyamoto S, Kasagi K, Mori T, Konishi J. Serial occurrence of two types of postpartum thyroid disorders. Usefulness of Tc-99m pertechnetate uptake. Clinical nuclear medicine. 1996;21(6):460462.

21. Nagai Y, Toya T, Fukuoka K, Tanaka N, Yanagi S, Kobayashi K. Occurrence and spontaneous remission of Graves' hyperthyroidism preceded by painless thyroiditis. Endocrine journal. 1997;44(6):881885.

22. Sarlis NJ, Brucker-Davis F, Swift JP, Tahara K, Kohn LD. Graves' disease following thyrotoxic painless thyroiditis. Analysis of antibody activities against the thyrotropin receptor in two cases. Thyroid. 1997;7(6):829-836.

23. Umena $\mathrm{S}$, Takano $\mathrm{T}$, Iijima $\mathrm{T}$, et al. A case of repeated painless thyroiditis followed by Graves' disease. Endocrine journal. 1995;42(6):821-826.

24. Kahaly GJ, Bartalena L, Hegedus L. The American Thyroid Association/American Association of Clinical Endocrinologists guidelines for hyperthyroidism and other causes of thyrotoxicosis: a European perspective. Thyroid. 2011;21(6):585-591.

25. Cappelli C, Pirola I, De Martino E, et al. The role of imaging in Graves' disease: a cost-effectiveness 


\section{Special Article}

analysis. European journal of radiology. 2008;65(1):99-103.

26. Costagliola S, Morgenthaler NG, Hoermann R, et al. Second generation assay for thyrotropin receptor antibodies has superior diagnostic sensitivity for Graves' disease. The Journal of clinical endocrinology and metabolism. 1999;84(1):90-97.

27. Hiraiwa $T$, Ito $M$, Imagawa $A$, et al. High diagnostic value of a radioiodine uptake test with and without iodine restriction in Graves' disease and silent thyroiditis. Thyroid. 2004;14(7):531-535.

28. Meller J, Becker W. The continuing importance of thyroid scintigraphy in the era of high-resolution ultrasound. European journal of nuclear medicine and molecular imaging. 2002;29 Suppl 2:S425-438.

29. Osaki Y, Sakurai K, Arihara Z, Hata M, Fukazawa H. Prediction of late (24-hour) radioactive iodine uptake using early (3-hour) uptake values in Japanese patients with Graves' disease. Endocrine Journal. 2012;59(2):173-7.

30. Hari Kumar KV, Pasupuleti V, Jayaraman M, Abhyuday V, Rayudu BR, Modi KD. Role of thyroid Doppler in differential diagnosis of thyrotoxicosis. Endocrine practice: official journal of the American College of Endocrinology and the American Association of Clinical Endocrinologists. 2009;15(1):6-9.

31. Ota H, Amino N, Morita S, et al. Quantitative measurement of thyroid blood flow for differentiation of painless thyroiditis from Graves' disease. Clinical endocrinology. 2007;67(1):41-45.

32. Erdogan MF, Anil C, Cesur M, Baskal N, Erdogan G. Color flow Doppler sonography for the etiologic diagnosis of hyperthyroidism. Thyroid. 2007;17(3):223-228.

33. Izumi Y, Hidaka Y, Tada H, et al. Simple and practical parameters for differentiation between destruction-induced thyrotoxicosis and Graves' thyrotoxicosis. Clinical endocrinology. 2002;57(1):51-58.

34. Sriphrapradang C, Bhasipol A. Differentiating Graves' disease from subacute thyroiditis using ratio of serum free triiodothyronine to free thyroxine. Annals of medicine and surgery. 2016; 10:69-72.

35. Yoshimura Noh J, Momotani N, Fukada S, Ito K, Miyauchi A, Amino N. Ratio of serum free triiodothyronine to free thyroxine in Graves' hyperthyroidism and thyrotoxicosis caused by painless thyroiditis. Endocrine journal. 2005;52(5):537-542.

36. Ilicki A, Gamstedt A, Karlsson FA. Hyperthyroid Graves' disease without detectable thyrotropin receptor antibodies. The Journal of clinical endocrinology and metabolism. 1992;74(5):1090-1094.

37. Morita T, Tamai H, Oshima A, et al. The occurrence of thyrotropin binding-inhibiting immuno- globulins and thyroid-stimulating antibodies in patients with silent thyroiditis. The Journal of clinical endocrinology and metabolism. 1990;71(4):10511055.

38. Avs AK, Mohan A, Kumar PG, Puri P. Scintigraphic Profile of Thyrotoxicosis Patients and Correlation with Biochemical and Sonological Findings. Journal of clinical and diagnostic research. 2017;11(5):Oc01-oc03.

39. Pearce EN, Hennessey JV, McDermott MT. New American Thyroid Association and American Association of Clinical Endocrinologists guidelines for thyrotoxicosis and other forms of hyperthyroidism: significant progress for the clinician and a guide to future research. Thyroid. 2011;21(6):573576.

40. Yamashita S, Amino N, Shong YK. The American Thyroid Association and American Association of Clinical Endocrinologists hyperthyroidism and other causes of thyrotoxicosis guidelines: viewpoints from Japan and Korea. Thyroid. 2011;21(6):577580.

41. Pearce EN, Farwell AP, Braverman LE. Thyroiditis. The New England journal of medicine. 2003;348(26):2646-2655.

42. Martino E, Bartalena L, Bogazzi F, Braverman LE. The effects of amiodarone on the thyroid. Endocrine reviews. 2001;22(2):240-254.

43. Martino E, Safran M, Aghini-Lombardi F, et al. Environmental iodine intake and thyroid dysfunction during chronic amiodarone therapy. Annals of internal medicine. 1984;101(1):28-34.

44. Bogazzi F, Bartalena L, Brogioni S, et al. Color flow Doppler sonography rapidly differentiates type I and type II amiodarone-induced thyrotoxicosis. Thyroid. 1997;7(4):541-545.

45. Popoveniuc G, Jonklaas J. Thyroid nodules. The Medical clinics of North America. 2012;96(2):329349.

46. Leger J, Olivieri A, Donaldson M, et al. European Society for Paediatric Endocrinology consensus guidelines on screening, diagnosis, and management of congenital hypothyroidism. The Journal of clinical endocrinology and metabolism. 2014;99(2):363-384.

47. Keller-Petrot I, Leger J, Sergent-Alaoui A, de Labriolle-Vaylet C. Congenital Hypothyroidism: Role of Nuclear Medicine. Seminars in nuclear medicine. 2017;47(2):135-142.

48. Silberstein EB. Radioiodine: the classic theranostic agent. Seminars in nuclear medicine. 2012;42(3):164-170. 